\title{
Traveling Waves in Relation to the Surface Fields on a Semi-Infinite Cone
}

\author{
T. B. A. Senior and P. H. Wilcox \\ Radiation Laboratory, Department of Electrical Engineering, University of Michigan, \\ Ann Arbor, Mich. 48103, U.S.A.
}

(Received December 14, 1966; revised January 5, 1967)

\begin{abstract}
For a plane electromagnetic wave at nose-on incidence on a semi-infinite perfectly conducting cone expressions for the surface field components are obtained. These have been programmed for numerical computation and data are presented for the magnitudes and phases as functions of the distance from the tip for three different cone angles. Comparison with results derived from the physical optics approximation and from a simple wedge approximation shows that the differences between the exact and physical optics values are not attributable to a tip-excited wave.
\end{abstract}

\section{The Genesis of the Problem}

Traveling waves have long played an important role in antenna theory. Their application to the theory of scattering by long thin bodies at oblique angles of incidence was realized a decade ago (Peters, 1956a,b), but in spite of this, the concept of such waves - their manner of excitation and the extent to which they can be regarded as self-sustaining wave motions - is not well understood.

As generally employed in scattering theory, a traveling wave is only a manifestation of the impressed effect of the incident field as long as the latter is present, and is therefore hardly identifiable with a form of surface wave within the illuminated region. With a long thin body, however, this same wave motion is assumed to persist into the shadow region with only a negligible change of character; and when the wave reaches the rear termination of the body, it can then be reflected to give a wave which travels in the backward direction and which is ultimately responsible for the field radiated in the back-scattering direction. That such a decomposition of the surface field is basically correct has been verified by detailed asymptotic analyses applied to the known exact solution for on-axis illumination of acoustically hard and soft prolate spheroids whose eccentricities are almost unity (Goodrich and Kazarinoff, 1963), and is also evident from the analytical treatments of scattering by thin wires, both finite (Einarsson and Tai, 1967) and semi-infinite (Vainshtein, 1959) in length.

The manner in which the surface field builds up as a function of distance away from the front end of the body is not apparent from the above studies, nor are we aware of any analysis which has yet thrown any light on this matter. On the other hand, a knowledge of the character and rapidity of the buildup could be quite important, not least from the point of view of designing an absorber which would effectively suppress the traveling wave. Thus, for example, with a long thin body whose radius is not a small fraction of the wavelength, the forward traveling wave is merely the physical optics current, and one could presumably design an absorber to attenuate this on the basis of a large flat plate or circular cylinder illuminated at an angle corresponding to that which the incident field makes with the appropriate generator of the body. But if the surface field requires some considerable distance to achieve the physical optics value, the design would be inappropriate over a significant fraction of the body's surface.

An attractive body on which to investigate this buildup is the semi-infinite cone illuminated by a plane wave at or near nose-on incidence. Probe measurements of the surface fields on cone-spheres for sufficiently large values of the base radius-towavelength ratio such that the fields on the sides of cone are relatively unaffected by the termination, have shown (Zukowski and Senior, 1965) that the fields do build up to values closely resembling those predicted by physical optics. But in the important region near to the tip, the measured data are substantially in error because of the sensitivity of the probe to lateral (azimuthal) variations in the field. Attention was therefore directed at the known analytical solution for the surface fields on a semi-infinite cone at nose-on incidence, and, for the first time, detailed computations of the infinite eigenfunction expansion have 
been performed. We shall here summarize both the analysis and the computed data with particular emphasis on the form and magnitude of the perturbation field associated with the tip, that is, on the difference between the exact and physical optics values of the surface fields.

\section{The Surface Field on a Semi-Infinite Cone}

Although solutions for some of the scattering problems associated with the semi-infinite cone have been known for almost a century, it was not until 1948 that Hansen and Schiff provided the first exact solution for the (vector) problem of a plane ei.ctromagnetic wave at nose-on incidence on a perfectiy conducting cone. Since then there have been a variety of analytical treatments appropriate to the more general types of dipole and plane-wave excitation (see, for example, Felsen, 1957), and it is sufficient to give here only a brief outline of one method of derivation which leads directly to expressions for the surface field components in the forms required for numerical computation.

In terms of the spherical polar coordinates $(r, \theta, \phi)$, which are related to the Cartesian coordinates $(x, y, z)$ by the equations

$$
\begin{aligned}
& x=r \sin \theta \cos \phi \\
& y=r \sin \theta \sin \phi \\
& z=r \cos \theta,
\end{aligned}
$$

the surface of the cone is defined as $\theta=\theta_{0}$. The exterior half-angle is therefore $\theta_{0}$, and the (interior) semivertex angle is $\alpha=\pi-\theta_{0}$.

A plane electromagnetic wave is incident in the direction of the negative $z$ axis. Without loss of generality its electric vector can be assumed to be in the $x$ direction and hence

$$
\underline{E}=\hat{x} e^{-i k z} \quad \underline{H}=-\hat{y} Y e^{-i k z},
$$

where $Y=1 / Z$ is the intrinsic admittance of free space and a time factor $e^{-i \omega t}$ has been suppressed. When expressed in terms of the spherical polar coordinates, the incident field becomes

$\underline{E}^{i}=\hat{r} \sin \theta \cos \phi+\hat{\theta} \cos \theta \cos \phi$

$$
-\hat{\phi} \sin \phi) e^{-i k r \cos \theta}
$$

$\underline{H}^{i}=-Y(\hat{r} \sin \theta \sin \phi+\hat{\theta} \cos \theta \sin \phi$

$$
+\hat{\phi} \cos \phi) e^{-i k r \cos \theta} \text {, }
$$

and the task now is to find the corresponding total (incident plus scattered) field $(\underline{E}, \underline{H})$ satisfying the boundary condition

$$
\hat{\theta} \times \underline{E}=0
$$

at the surface $\theta=\theta_{0}$ of the perfectly conducting cone.

To this end we introduce two Debye potentials $u$ and $v$, and write the components of the total field as

$$
\begin{aligned}
& E_{r}=\frac{\partial^{2}}{\partial r^{2}}(r u)+k^{2} r u \\
& E_{\theta}=\frac{1}{r} \frac{\partial^{2}}{\partial r \partial \theta}(r u)+\frac{i k Z}{r \sin \theta} \frac{\partial}{\partial \phi}(r v) \\
& E_{\phi}=\frac{1}{r \sin \theta} \frac{\partial^{2}}{\partial r \partial \phi}(r u)-\frac{i k Z}{r} \frac{\partial}{\partial \theta}(r v) \\
& H_{r}=\frac{\partial^{2}}{\partial r^{2}}(r v)+k^{2} r v \\
& H_{\theta}=\frac{1}{r} \frac{\partial^{2}}{\partial r \partial \theta}(r v)-\frac{i k Y}{r \sin \theta} \frac{\partial}{\partial \phi}(r u) \\
& H_{\phi}=\frac{1}{r \sin \theta} \frac{\partial^{2}}{\partial r \partial \phi}(r u)+\frac{i k Y}{r} \frac{\partial}{\partial \theta}(r u) .
\end{aligned}
$$

By virtue of the geometry and the form of the incident field, natural representations for $u$ and $v$ are

$$
\begin{aligned}
& u=\sum a_{\nu j_{\nu}}(k r) P_{\nu}^{1}(\cos \theta) \cos \phi \\
& v=\sum b_{\mu} j_{\mu}(k r) P_{\mu}^{1}(\cos \theta) \sin \phi
\end{aligned}
$$

in which $j_{s}(k r)$ is the spherical Bessel function of order $s$ and

$$
P_{i s}^{1}(\cos \theta) \equiv \frac{\partial}{\partial \theta} P_{s}(\cos \theta)
$$

is the associated Legendre function of degree $s$ and order unity as defined, for example, by Magnus and Oberhettinger (1949). The coefficients $a_{\nu}$ and $b_{\mu}$, together with the ranges of the summation variables $\nu$ and $\mu$, are as yet unspecified, but from an examination of (4) it is seen that if $\nu$ is chosen to be a zero of $P_{s}^{1}\left(\cos \theta_{0}\right)$, that is $\nu=\nu_{n}, n=1,2,3, \ldots$. with

$$
P_{\nu_{n}}^{1}\left(\cos \theta_{0}\right)=0 \text {, }
$$

and if $\mu$ is chosen to be a zero of

$$
\frac{\partial}{\partial \theta_{0}} P_{s}^{1}\left(\cos \theta_{0}\right)
$$

that is, $\mu=\mu_{n}, n=1,2,3, \ldots$ with

$$
\frac{\partial}{\partial \theta_{0}} P_{\mu_{n}}^{1}\left(\cos \theta_{0}\right)=0 \text {, }
$$

the boundary condition (3) is fulfilled, and the summations in (5) and (6) then extend over all the positive roots $\nu_{n}$ and $\mu_{n}$ of (7) and (8) respectively. 
The coefficients $a_{v}$ and $b_{\mu}$ are determined by expanding the incident field (3) in a form analogous to (5) and (6), using the orthogonality of the functions $P_{\nu_{n}}^{1}(\cos \theta)$ and $P_{\mu_{n}}^{1}(\cos \theta)$ over the interval $\left(0, \theta_{0}\right)$, and matching the resulting coefficients with those in (5) and (6). It is found that (Mentzer, 1955)

$$
\begin{gathered}
a_{\nu_{n}}=-\frac{2 i}{k \sin \theta_{0}} \frac{\left(2 \nu_{n}+1\right) e^{-i \nu_{n} \frac{\pi}{2}}}{\frac{\partial}{\partial \nu_{n}} P_{\nu_{n}}^{1}\left(\cos \theta_{0}\right) \frac{\partial}{\partial \theta_{0}} P_{\nu_{n}}^{1}\left(\cos \theta_{0}\right)} \\
b_{\mu_{n}}=\frac{2 i Y}{k \sin \theta_{0}} \frac{\left(2 \mu_{n}+1\right) e^{-i \mu_{n} \frac{\pi}{2}}}{\frac{\partial^{2}}{\partial \mu_{n} \partial \theta_{v}} P_{u_{n}}^{1}\left(\cos \theta_{0}\right)}
\end{gathered}
$$

which now complete the specification of the total field. On the surface of the cone the only nonzero components are $E_{\theta}, H_{r}$ and $H_{\phi}$, and after some simplification the expressions for these can be written as

$$
\begin{aligned}
& E_{\theta}=\frac{\cos \phi}{\rho}\left\{i \frac{\partial \Lambda}{\partial \theta_{0}}+\frac{\Lambda^{\prime}}{\sin \theta_{0}}\right\} \\
& H_{r}=-i Y \sin \phi\left(\frac{\partial^{2}}{\partial \rho^{2}}+1\right) \Lambda^{\prime} \\
& H_{\phi}=-Y \frac{\cos \phi}{\rho}\left\{\frac{i}{\sin \theta_{0}} \frac{\partial \Lambda^{\prime}}{\partial \rho}+\Lambda\right\},
\end{aligned}
$$

where

$$
\begin{gathered}
\Lambda=-\frac{2}{\sin \theta_{0}} \sum_{n}\left(2 \nu_{n}+1\right) \frac{e^{-i \nu_{n} \frac{\pi}{2}} \psi_{\nu_{n}}(\rho)}{\frac{\partial}{\partial \nu_{n}} P_{\nu_{n}}^{1}\left(\cos \theta_{0}\right)} \\
\Lambda^{\prime}=\frac{2}{\sin \theta_{0}} \sum_{n}\left(2 \mu_{n}+1\right) \frac{e^{-i \mu_{n} \frac{\pi}{2}} \psi_{\nu_{n}}(\rho)}{\frac{\partial^{2}}{\partial \mu_{n} \partial \theta_{0}} P_{\mu_{n}}^{1}\left(\cos \theta_{0}\right)}
\end{gathered}
$$

and, for convenience, we have put

$$
\rho=k r \quad \psi_{s}(\rho)=\rho j_{s}(\rho) .
$$

We note in passing that

$$
\left(\frac{\partial^{2}}{\partial \rho^{2}}+1\right) \psi_{s}(\rho)=\frac{s(s+1)}{\rho^{2}} \psi_{s}(\rho) .
$$

The expressions for the three surface field components shown in (11) were the effective starting point for the present study. Each is a function of $\rho, \theta_{0}$ and $\phi$, but since the $\phi$ dependence is explicit, it can be suppressed by writing

$$
\begin{aligned}
& E_{\theta}=\cos \phi \mathscr{E}_{\theta} \\
& H_{r}=Y \sin \phi \mathscr{H}_{r} \\
& H_{\phi}=Y \cos \phi \mathscr{H}_{\phi} .
\end{aligned}
$$

The actual formulae for $\mathscr{E}_{\theta}, \mathscr{H}_{r}$ and $\mathscr{H}_{\phi}$ are evident from (11) and the task that now confronts us is the computation of these as functions of $\rho$ for selected values of $\theta_{0}$.

\section{Computational Procedures}

The heart of the computation of $\mathscr{E}_{\theta}, \mathscr{H}_{r}$ and $\mathscr{H}_{\phi}$ is the calculation of the zeros of the Legendre function and its first derivative, that is, the determination of the two sets of values $\nu_{n}$ and $\mu_{n}, n=1,2,3, \ldots$, satisfying (7) and (8) for a prescribed $\theta_{0}$. Although this problem has received quite some attention over the years, most of the work was carried out before the advent of large, high-speed digital computers, and neither approximate analyses nor hand computations have produced values for the zeros accurate enough for use in the calculation of expressions such as those for $\Lambda$ and $\Lambda^{\prime}$ (see, for example, Carrus and Treuenfels, 1950; Siegel et al., 1952, 1953). An exception to this is the more recent work of Waterman (1963) in which zeros were obtained to a stated accuracy of seven significant figures by numerical integration of the Mehler-Dirichlet representation of the Legendre function, and we shall comment upon these results in a moment.

The representation which formed the basis for the present computation of the zeros is the trigonometric expansion

$P_{j}^{i}(\cos \theta)=\pi^{-1 / 2} 2^{i+1}(\sin \theta)^{i}$

$$
\begin{aligned}
\times \frac{\Gamma(i+j+1)}{\Gamma(j+3 / 2)} \sum_{k=0}^{\infty} \frac{(i+1 / 2)_{k}(i+j+1)_{k}}{k !(j+3 / 2)_{k}} & \\
\times & \sin \{(1+j+2 k+1) \theta\}
\end{aligned}
$$

(NBS, 1964), valid for $0<\theta<\pi$. If $i=-1$, the convergence of the series is improved, and since

$$
P_{j}^{1}(\cos \theta)=-\frac{\Gamma(j+2)}{\Gamma(j)} P_{j}^{-1}(\cos \theta),
$$

an expansion for the Legendre function of order unity is

$$
P_{j}^{1}(\cos \theta)=-\frac{1}{\sqrt{\pi} \sin \theta} \frac{\Gamma(j+2)}{\Gamma(j+3 / 2)} S_{j}(\theta)
$$

with $\quad S_{j}(\theta)=\sum_{k=0}^{\infty} \frac{\left(-\frac{1}{2}\right)_{k}()_{k}}{k !(j+3 / 2)_{k}} \sin \{(j+2 k) \theta\}$.

Similarly, from the recurrence relation

$$
\left(z^{2}-1\right) \frac{\partial}{\partial \dot{z}^{\prime}} P_{j}^{1}(z)=j z P_{j}^{1}(z)-(j+1) P_{j-1}^{1}(z)
$$

with $z=\cos \theta$, an expansion for the derivative of the 
Legendre function is

$$
\begin{aligned}
\frac{\partial}{\partial \theta} P_{j}^{1}(\cos \theta)= & -\frac{1}{\sin ^{2} \theta} \frac{\Gamma(j+2)}{\Gamma(j+3 / 2)} \\
& \times_{j}\left\{\cos \theta S_{j}(\theta)-\left(1+\frac{1}{2 j}\right) S_{j-1}(\theta)\right\}
\end{aligned}
$$

and the required zeros $\nu_{n}$ and $\dot{\mu}_{n}$ for a specified $\theta=\theta_{0}$ are now obtained from (16) and (18) respectively by successive iteration based on Newton's method.

Although the relatively slow convergence of the series for $S_{j}(\theta)$ limits the accuracy with which it can be computed, no difficulty has been found in achieving values for the zeros which are believed accurate to 7 or 8 significant figures, with the accuracy decreasing towards the lower number as the order of the zero increases, but otherwise not significantly affected by the value of $\theta_{0}$. Comparison with the results that Waterman (1963) has provided for the case $\theta_{0}=165^{\circ}$, shows agreement to within unity in the 7th significant figure for the zeros of the Legendre function, but for the zeros of the derivative, the results for all zeros beyond the first are consistently larger than those of Waterman in the 6th and 7th significant figure. In spite of several checks which have been applied to the program, no explanation for the discrepancy has been found. At present the iteration for the zeros is continued until there is no change in the 8th decimal place, with the program computing $S_{j}\left(\theta_{0}\right)$ out to the point at which the last term in the series is less than $10^{-8}$, or until 500 terms have been included, whichever occurs first. In general 500 terms were included, with the magnitude of the last term increasing with the order of the zero, but tests run with an iteration tolerance of $10^{-10}$ and 1000 terms retained in the series (leading to a decrease of one order of magnitude in the size of the last term), showed that the original values of the zeros were good to at least 7 (and often 8) significant figures. The factor limiting the precision of the results at this point is the error caused by the truncation of the series $S_{j}\left(\theta_{0}\right)$.

Once the zeros $\nu_{n}$ and $\mu_{n}$ have been determined, the evaluation of the expressions for the surface field components is relatively straightforward. Since the denominator of each term in the expansions for $\Lambda$ and $\Lambda^{\prime}$ arises naturally in the course of the iteration for the zeros, all such factors not involving $\rho$ are combined and computed along with each zero. The expansion used for the function $\psi_{s}(\rho)$ is derived from that of the cylindrical Bessel function, and is

$$
\psi_{s}(\rho)=\sqrt{\pi} \frac{\left(\frac{\rho}{2}\right)^{s+1}}{(s+3 / 2)} \sum_{k=0}^{x} \frac{\left(-\frac{\rho^{2}}{4}\right)^{k}}{k !(s+3 / 2)_{k}}
$$

To avoid undue loss of accuracy in the computation, (19) is employed only when $s$ is not small compared with $\rho$, and in the remaining cases, the function is evaluated for a much larger value of the order, followed by a repeated application of the recurrence relation

$$
\psi_{s-1}(\rho)=\frac{2 s+1}{\rho} \psi_{s}(\rho)-\psi_{s+1}(\rho)
$$

to obtain the required function accurate to 7 decimals. In the final summations to compute the series expressions (12) and (13) for $\Lambda$ and $\Lambda^{\prime}$ respectively, the number $N$ of terms retained has been somewhat arbitrarily set at $N=3 p+5$, rounded up to the next integer. Examination of the truncation error at this point showed it to be much less than any error involved in the determination of values for $\psi_{s}(\rho)$.

\section{The Behavior of the Surface Field Components}

Before examining the results of the digital computation, a few remarks about the expected form of the surface field components would appear to be in order.

In the immediate vicinity of the tip, the behavior of $\mathscr{E}_{\theta}, \mathscr{H}_{r}$ and $\mathscr{H}_{\phi}$ is determined by the first (and smallest) zeros $\nu_{1}$ and $\mu_{1}$. If $\theta_{0}<\pi, \nu_{1}$ exceeds unity, and some typical values of $\nu_{1}$ and $\nu_{2}$ for $\pi / 2 \leqslant \theta_{0}<\pi$ are as follows:

$$
\begin{array}{lllll} 
& \theta_{0}=90^{\circ} & \theta_{0}=150^{\circ} & \theta_{0}=165^{\circ} & \theta_{0}=172^{1 / 2^{\circ}} \\
\nu_{1} & 2.0000 & 1.1156 & 1.0316 & 1.0083 \\
\nu_{2} & 4.0000 & 2.2780 & 2.0844 & 2.0237 .
\end{array}
$$

On the other hand, $\mu_{1}$ is less than unity for $\pi / 2<\theta_{0}$ $<\pi$, and some typical values of $\mu_{1}$ and $\mu_{2}$ are:

$$
\begin{array}{lllll} 
& \theta_{0}=90^{\circ} & \theta_{0}=150^{\circ} & \theta_{0}=165^{\circ} & \theta_{0}=172^{1 / 2^{\circ}} \\
\mu_{1} & 1.0000 & 0.9005 & 0.9671 & 0.9914 \\
\mu_{2} & 3.0000 & 1.8857 & 1.9189 & 1.9748 .
\end{array}
$$

The variation of $\mu_{1}$ as a function of $\theta_{0}$ for the range $2 \frac{1}{2}{ }^{\circ} \leqslant \theta_{0} \leqslant 177^{1 / 2^{\circ}}$ is shown in figure 1 .

From an examination of (11) through (15) it is apparent that for $\pi / 2<\theta_{0}<\pi$ the tip behavior of the component $\mathscr{C}_{\theta}$ is determined by the value of $\nu_{1}$ alone, whereas $\mu_{1}$ determines the behavior of $\mathscr{H}_{r}$ and $\mathscr{H}_{\phi}$. Explicitly

$$
\mathscr{E}_{\theta}=O\left(\rho^{\nu_{1}-1}\right) ; \quad \mathscr{H}_{r}, \mathscr{H}_{\phi}=O\left(\rho^{\mu_{1}-1}\right),
$$

and thus $\mathscr{H}_{r}$ and $\mathscr{H}_{\phi}$ become infinite as $\rho \rightarrow 0$ while $\mathscr{E}_{\theta}$ tends to zero. However, since $\left|\mu_{1}-1\right| \ll 1$, the region in which the tip behavior is dominant will be confined to the immediate vicinity of the tip, and this will be true also of the buildup of $\mathscr{E}_{A}$ from zero if $\theta_{0}$ is not too different from $\pi$. 


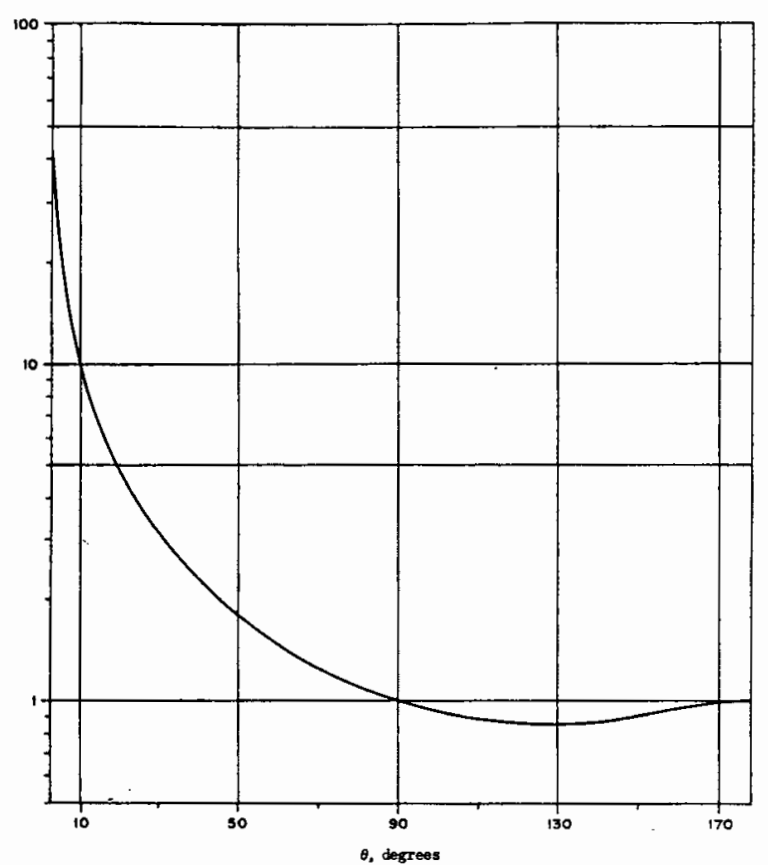

FigURE 1. First zero $\mu=\mu_{1}$ of $\frac{\partial}{\partial \theta} P_{\mu}^{1}(\cos \theta)$.

At sufficiently large distances from the tip, the physical optics approximation is expected to provide an accurate picture of the surface field behavior. Although the approximation is generally used only to specify the tangential components of the total magnetic field, the same conceptual basis can be used to predict the normal electric field, and there is no obvious reason why the prediction should not be of comparable accuracy to the estimates of the tangential magnetic field. We thus obtain

$$
\begin{aligned}
& \mathscr{E}_{\theta}^{\text {p.o. }}=2 \cos \theta_{0} e^{-i \rho \cos \theta_{0}} \\
& \mathscr{H}_{r}^{\text {p.o. }}=-2 \sin \theta_{0} e^{-i \rho \cos \theta_{0}} \\
& \mathscr{X}_{\phi}^{\text {p.o. }}=-2 e^{-i p \cos \theta_{0}},
\end{aligned}
$$

and some values for the moduli of the optics approximations to the field components are then:

$\begin{array}{cccc} & \theta_{0}=150^{\circ} & \theta_{0}=165^{\circ} & \theta_{0}=172^{1 / 2^{\circ}} \\ \left|\mathscr{E}_{\theta}^{\text {p.o. }}\right| & 1.7233 & 1.9273 & 1.9829 \\ \left|\mathscr{H}_{+}^{\text {p.o. }}\right| & 1.0000 & 0.5176 & 0.2611 \\ \left|\mathscr{H}_{\phi}^{\text {p.o. }}\right| & 2.0000 & 2.0000 & 2.0000 .\end{array}$

For values of $\rho$ which are not so large that the physical optics approximation is appropriate, we can gather some information about the probable behavior of the surface field components by considering the corresponding two-dimensional problem of a perfectly conducting wedge excited by a plane wave at symmetrical incidence. For wedges of relatively small included angle, the fields on each surface are, to a first approximation, the same as on a half-plane inclined at the appropriate angle to the field, and by choosing the polarization of the incident field in such a way as to yield components analogous to those of interest in the cone problem, the following estimates for the surface fields on a cone are arrived at:

$$
\begin{aligned}
& \mathscr{L}_{\theta}^{w}=2 \cos \theta_{0} e^{-i p \cos \theta_{0}}\left[1-\frac{e^{-i \frac{\pi}{4}}}{\pi}\right. \\
& \left.\times\left\{F(\tau)-\frac{i}{2 \tau}\left(1+\sec \theta_{0}\right) e^{i \tau^{2}}\right\}\right] \\
& \mathscr{H}_{r}^{w}=-2 \sin \theta_{0} e^{-i p \cos \theta_{0}}\left[1-\frac{e^{-i \frac{\pi}{4}}}{\pi}\left\{F(\tau)-\frac{i}{2 \tau} e^{i \tau^{2}}\right\}\right] \\
& \mathscr{H}_{\phi}^{\mathcal{W}}=-2 e^{-i p \cos \theta_{0}}\left[1-\frac{e^{-i \frac{\pi}{4}}}{\pi} F(\tau)\right] .
\end{aligned}
$$

The affix " $w$ " here denotes the wedge approximation and $F(\tau)$ is the Fresnel integral

$$
F(\tau)=\int_{\tau}^{\infty} e^{i \lambda 2} d \lambda
$$

with

$$
\tau=\sqrt{2 \rho} \cos \frac{\theta_{0}}{2} \text {. }
$$

Since

$$
F(\tau) \sim \frac{i}{2 \tau} e^{i \tau^{2}}
$$

for large $\tau$, it is obvious that each of the field components (23) approaches its physical optics value (22) at sufficient distances from the tip, but for $\rho$ less than this all three components show an oscillatory behavior. This is illustrated in figures 2 and 3 in which the moduli and phases of the wedge approximations (23) are plotted as functions of $\rho$ for $\theta_{0}=150^{\circ}$. For very small values of $\rho$ the behavior of the wedge approximation is dictated by the edge condition at a two-dimensional singularity, namely

$$
\mathscr{E}_{\theta}^{w}, \mathscr{H}_{F}=O\left(\rho^{-1 / 2}\right) ; \quad \mathscr{H}_{\phi}^{w}=O\left(\rho^{1 / 2}\right),
$$

and since these variations are quite distinct from those appropriate to a tip (see (21)), it is apparent that the wedge approximation will not be applicable in the immediate vicinity of the tip. For other values of $\rho$, however, the wedge approximation does reproduce the essential features of the surface field behavior on the cone and, as we shall see shortly, the estimates given in (23) are accurate to a surprisingly high degree. This is in spite of the usual quantitative discrepancy between solutions of two- and three-dimensional prob- 


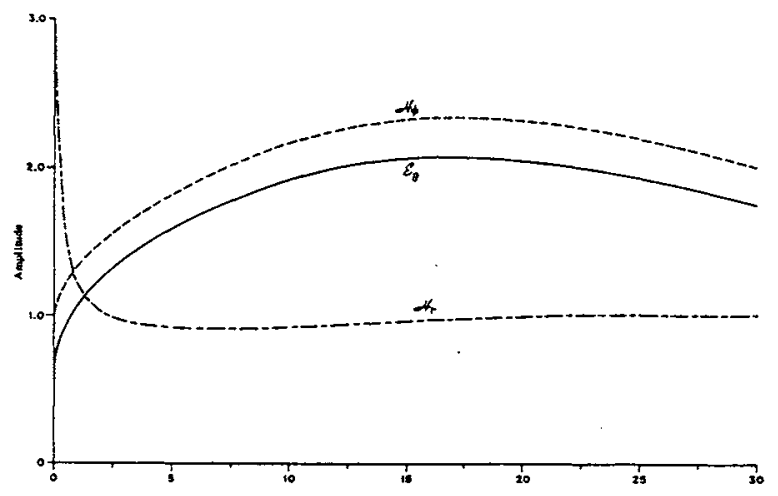

Figure 2. Amplitudes of surface field components $\mathscr{E}_{\theta}, \mathscr{H}_{r}$ and $\mathscr{X}_{\phi}$ for $\alpha=30^{\circ}$, derived from wedge approximation (4.23).

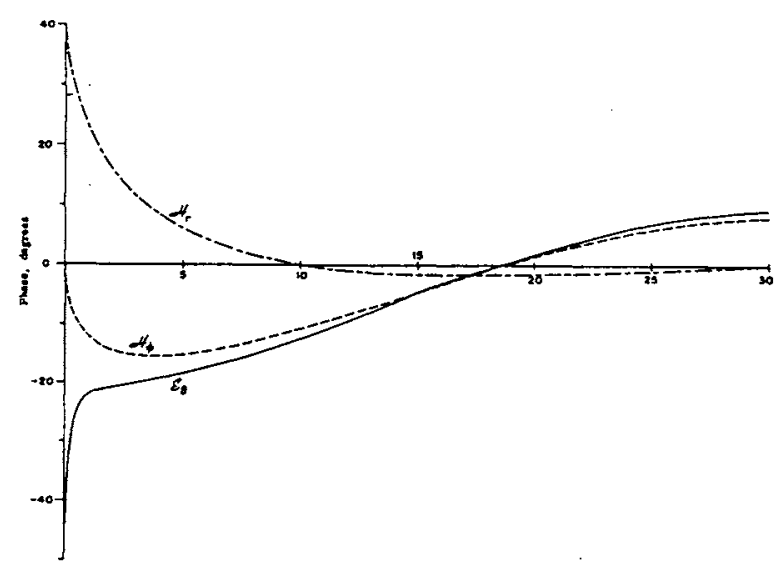

FIGURE 3. Phases of surface field components $\mathscr{E}_{\theta}, \mathscr{H}_{r}$ and $\mathscr{K}_{\phi}$, relative to those of physical optics field (4.22), for $\alpha=30^{\circ}$, derived from wedge approximation (4.23).

lems resulting from the differing dependences on distance.

\section{Numerical Results}

Using the computational techniques described in section 3 , the three surface field components $\mathscr{E}_{\theta}, \mathscr{H}_{r}$, and $\mathscr{H}_{\phi}$ were computed in real and imaginary parts, amplitude and phase, as functions of $\rho, 0<\rho \leqslant 30$, for a sequence of values of $\theta_{0}$ spanning the range $150^{\circ} \leqslant \theta_{0} \leqslant 172 \frac{1}{2^{\circ}}$. The computations were carried out on the IBM 7090 at the University of Michigan, and the results for $\theta_{0}=150^{\circ}, 165^{\circ}$, and $172^{1 / 2^{\circ}}$, corresponding to cones of half-angle $\alpha=30^{\circ}, 15^{\circ}$, and $71 / 2^{\circ}$ respectively, are presented here with the amplitudes shown in figures 4,6 , and 8 , and the phases (relative to those of the physical optics approximation (22)) given in figures 5,7 , and 9.

Starting first with the case $\alpha=30^{\circ}$, we observe that

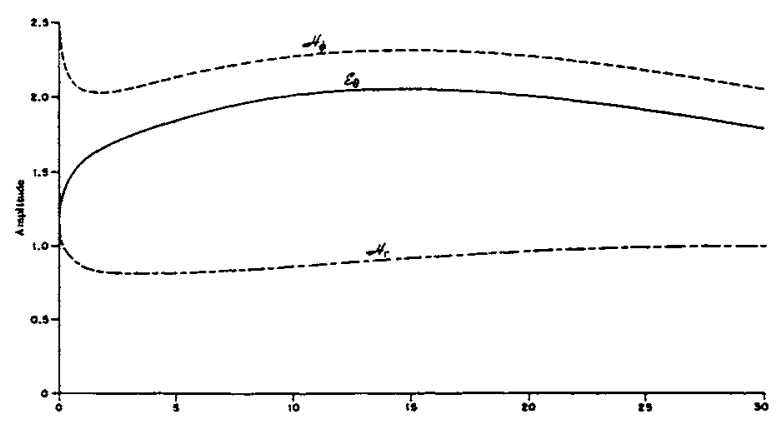

Figure 4. Amplitudes of surface field components $\mathscr{E}_{\theta}, \mathscr{H}_{r}$ and $\mathscr{H}_{\phi}$ for $\alpha=30^{\circ}$.

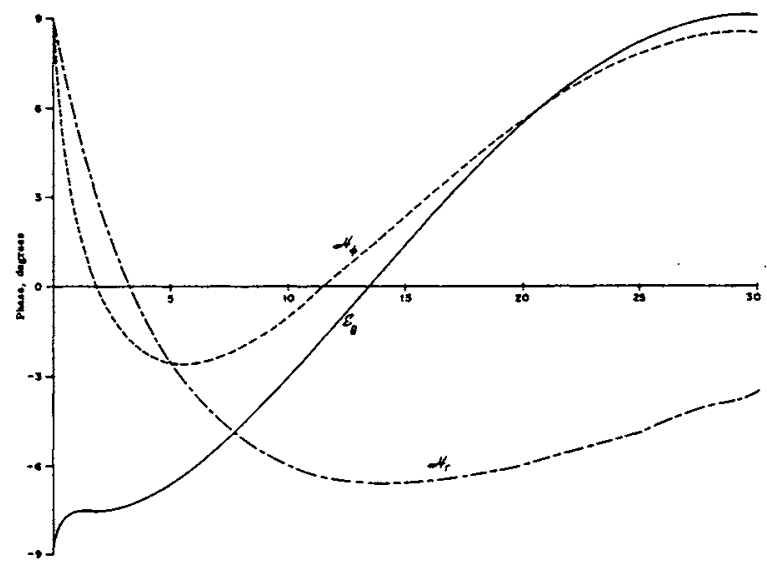

Figure 5. Phases of surface field components $\mathscr{E}_{\theta}, \mathscr{X}_{r}$ and $\mathscr{K}_{\phi}$ relative to those of physical optics field (4.22), for $\alpha=30^{\circ}$.

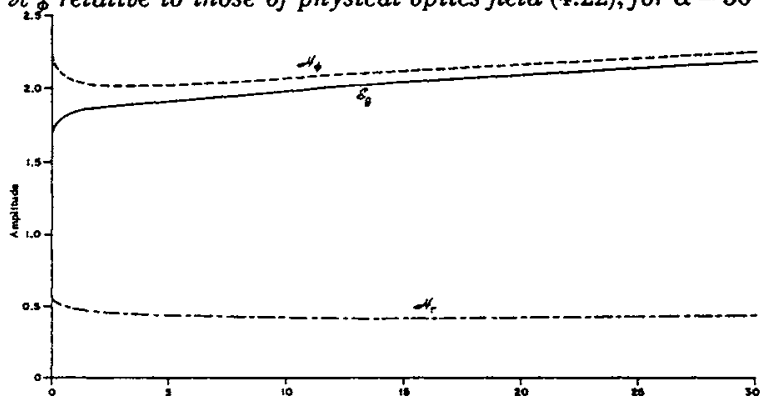

Figure 6. Amplitudes of surface field components $\mathscr{E}_{\theta}, \mathscr{X}_{T}$ and $\mathscr{H}_{\phi}$ for $\alpha=15^{\circ}$.

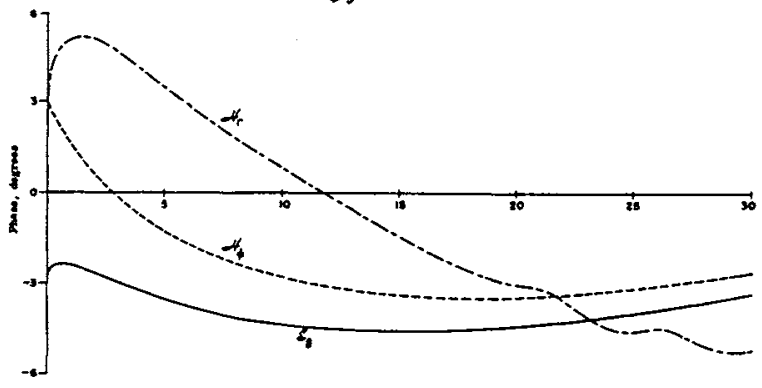

Figure 7. Phases of surface field components $\mathscr{L}_{\theta}, \mathscr{H}_{r}$ and $\mathscr{H}_{\phi}$, relative to those of physical optics field (4.22), for $\alpha=15^{\circ}$. 


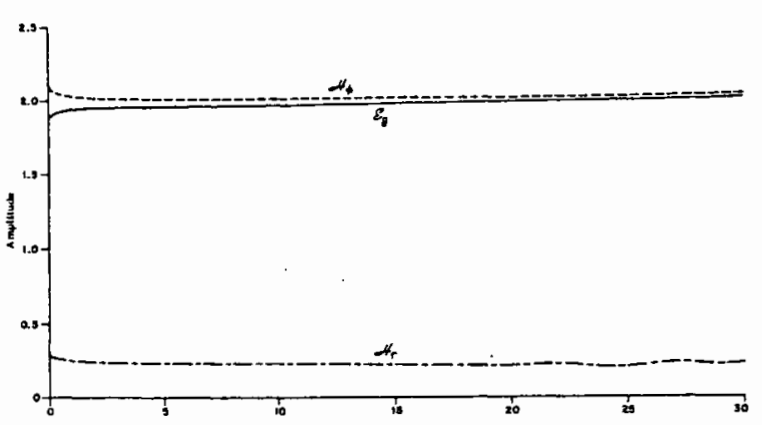

Figure 8. Amplitudes of surface field components $\mathscr{E}_{\theta}, \mathscr{H}_{r}$ and $\mathscr{H}_{\phi}$ for $\alpha=712^{\circ}$.

the region where the tip behavior dominates the surface field amplitude is confined to within $\lambda / 2$ (approx.) of the tip. Thereafter, $\left|\mathscr{H}_{r}\right|$ would appear to approach its physical optics value more or less asymptotically from below, and has almost reached that value when $\rho=30$, whereas $\left|\mathscr{H}_{\phi}\right|$ and $\left|\mathscr{E}_{\theta}\right|$ oscillate about their limiting values. For all three components, the behavior is strikingly similar to that revealed by the wedge approximation, and a comparison of figures 2 and 4 shows that this approximation is accurate to within 5 percent for $\rho>10$. The residual phases of the three components when the physical optics phases are subtracted are less than $10^{\circ}$ at all points more than about $0.01 \lambda$ from the tip, and are once again predicted to a very large extent by the wedge approximation (see fig. 3). For the component $\mathscr{H}_{r}$, however, the agreement is somewhat poorer than for the amplitude, and the discrepancy is of order $4^{\circ}$ for $r>2 \lambda$. We also note the slight oscillation of the phase of $\mathscr{H}_{r}$ that is evident at distances of more than about $3 \lambda$. Such oscillations are intensified when the cone angle $\alpha$ is reduced, and since detailed checks of the computational procedures have revealed no errors of this magnitude, it must be concluded that the oscillations are real.

At the next smaller value of $\alpha, \alpha=15^{\circ}$, the region where the tip behavior dominates the amplitude is still more reduced and, for example, $\left|\mathscr{H}_{r}\right|$ is still only a factor of two greater than its optics limit at a distance of $10^{-10} \lambda$ from the tip in spite of the fact that it must become infinite as $r \rightarrow 0$ in accordance with the condition (21). Away from the tip, $\left|\mathscr{H}_{r}\right|$ again approaches its physical optics value more quickly than the other two components, which are now somewhat closer in magnitude and oscillate about their optics limits with a period of oscillation which is much greater than for $\alpha=30^{\circ}$. Such changes as a function of $\alpha$ are fully predicted by the wedge approximation (23). The phases for $\alpha=15^{\circ}$ (see fig. 7) are within $6^{\circ}$ of their physical optics values throughout the range of $\rho$ computed, and the oscillation noted in the phase of $\mathscr{H}_{r}$ for $r>2 \lambda$ when $\alpha=30^{\circ}$ is here more well developed, with a trace of it also being detectable in the numerical data for the amplitude.

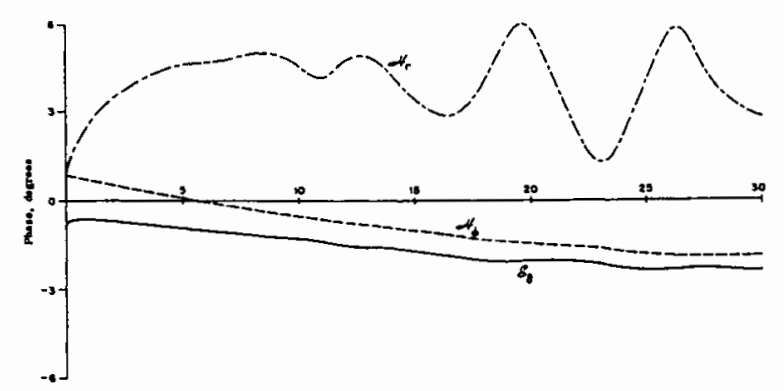

FigURE 9. Phases of surface field components $\mathscr{E}_{\theta}, \mathscr{H}_{r}$ and $\mathscr{H}_{\phi}$, relative to those of physical optics field (4.22), for $\alpha=7 \frac{1}{2^{\circ}}$.

At the smallest value of $\alpha$ considered, $\alpha=71 / 2^{\circ}$, the results are shown in figures 8 and 9 . Because of the vastly increased period of the main oscillation of the amplitudes, the amplitudes of the three components are almost constant out to the largest $\rho$ computed with the exception of the immediate vicinity of the tip. The small period oscillation of $\left|\mathscr{H}_{r}\right|$ is now evident in the plot, and this is reflected in a marked variation of its phase (see fig. 9). The oscillation commences near $\rho=5$ and appears to achieve a maximum amplitude near $\rho=23$, decreasing thereafter. Because of this, the phases depart from their physical optics values by almost as much as for $\alpha=15^{\circ}$, and we also observe from figure 9 that the phase of $\mathscr{E}_{\theta}$ is oscillating to some extent.

In order to throw some light on the nature of the discrepancy between the exact and physical optics values for the surface field components, the differences between the exact results and the physical optics estimates (22) have been computed for the same three values of $\alpha$. The amplitudes of the difference (or "perturbation") fields are shown in figures 10, 12, and 14 , and as expected they are comparatively small except within the immediate vicinity of the tip. They also decrease with decreasing $\alpha$, and in so doing, the small period oscillations, which are only just apparent in the curves for the complete surface field amplitudes, become more pronounced.

Of more interest, however, are the phases of the perturbed fields plotted in figures 11,13 , and 15 . For ease of presentation, the phases have been modified by subtracting those of the physical optics approximation (22), but such a modification is by no means sufficient to account for most of the variation of the phases of the perturbation fields with $\rho$. Although the phases of the complete surface field components are quite closely approximated by the physical optics phase, this is not true of the perturbation field, and accordingly this field cannot be explained by a simple "scaling" of the physical optics estimates. Nor is the phase of the perturbation field compatible with that of a spherical wave diverging from the tip, so that an interpretation in terms of a tip-excited correction is also inappropriate. 


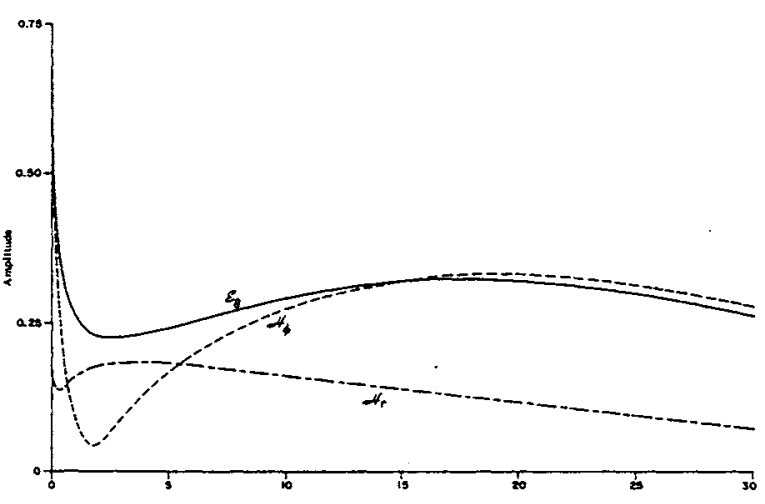

FigURE 10. Amplitudes of components $\mathscr{E}_{\theta}, \mathscr{H}_{r}$ and $\mathscr{H}_{\phi}$ of the perturbation field for $\alpha=30^{\circ}$.

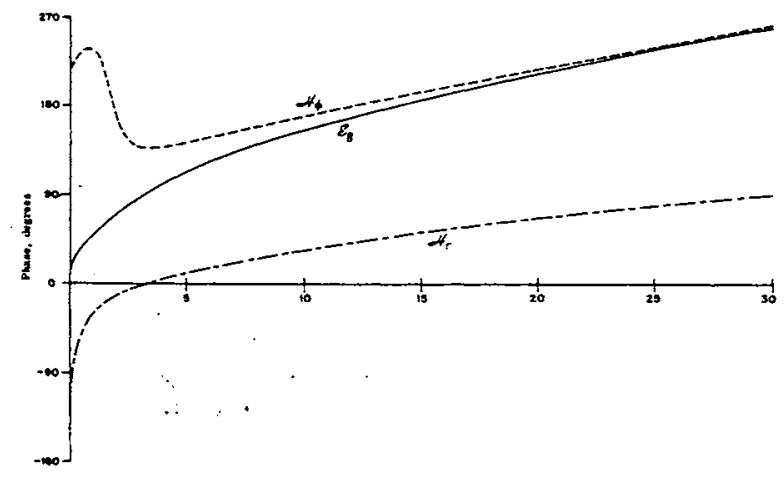

Ficure Il. Phases of components $\mathscr{E}_{\theta}, \mathscr{H}_{r}$ and $\mathscr{H}_{\phi}$, relative to the physical optics phases, for the perturbation field with $\alpha \stackrel{p}{=} 30^{\circ}$.

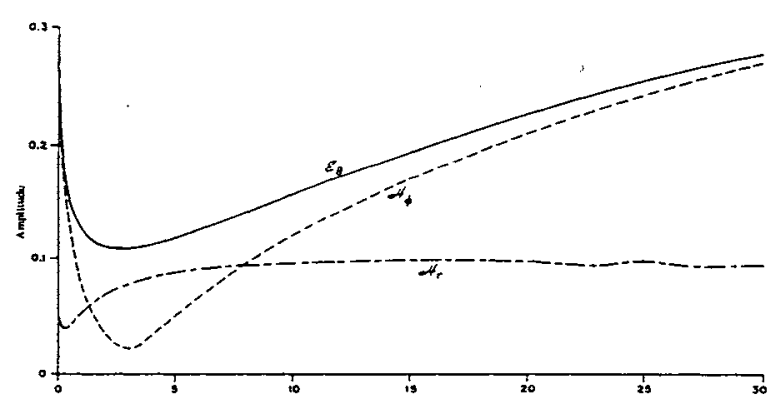

Figure 12. Amplitudes of components $\mathscr{E}_{\theta}, \mathscr{H}_{r}$ and $\mathscr{H}_{\phi}$ of the perturbation field for $\alpha=15^{\circ}$.

The failure of both these explanations to account for the phases in figures 11,13 , and 15 is no longer surprising when we recall the agreement between the estimates (23) based on the wedge approximation and the exact data for the surface field components at distances of more than $2 \lambda$ or so from the tip. The functional behavior of the wedge approximation is governed by the Fresnel integral, and the physical origin of this integral lies in the proximity of the surface to the reflected wave boundary at which the physical optics field is discontinuous, necessitating

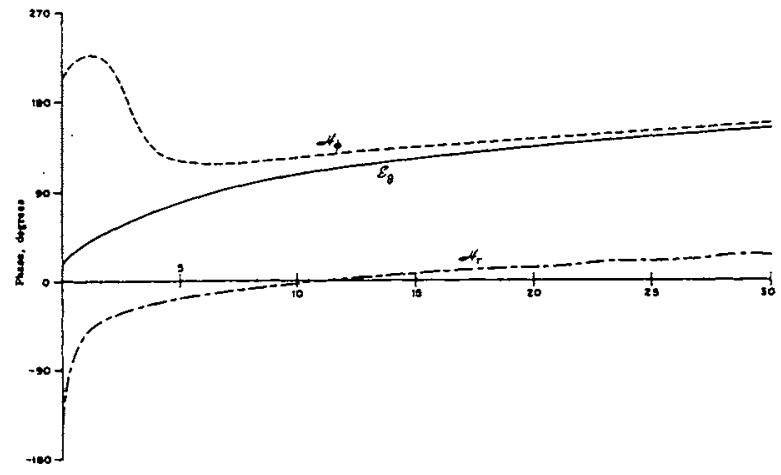

FIGURE 13. Phases of components $\mathscr{E}_{\theta}, \mathscr{H}_{r}$ and $\mathscr{H}_{\phi}$, relative to the physical optics phases, for the perturbation field with $\alpha=15^{\circ}$.

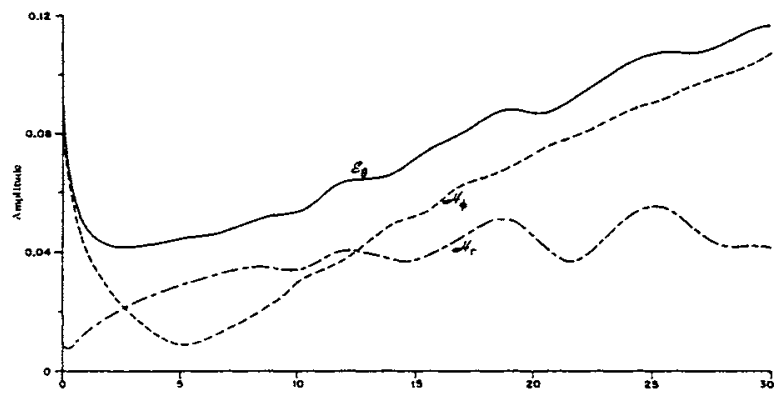

FIGURE 14. Amplitudes of components $\mathscr{E}_{\theta}, \mathscr{H}_{r}$ and $\mathscr{H}_{\phi}$ of the perturbation field for $\alpha=7 \frac{1}{1} 2^{\circ}$.

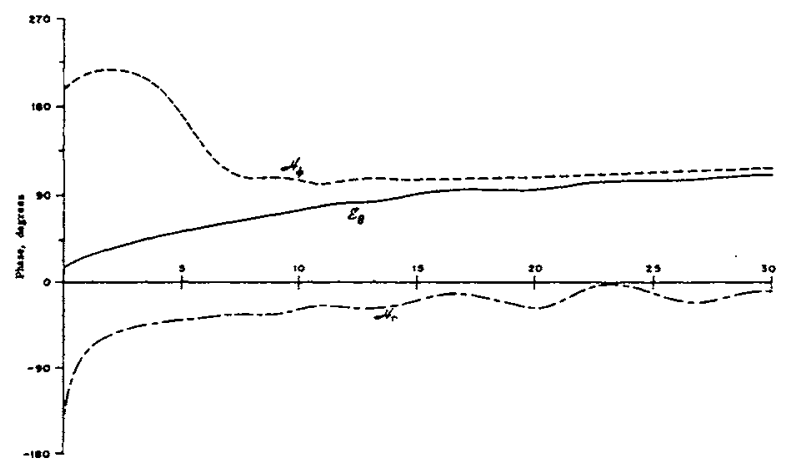

Figure 15. Phases of components $\mathscr{E}_{B}, \mathscr{H}_{r}$ and $\mathscr{H}_{\phi}$ relative to the physical optics phases, for the perturbation field with $\alpha=71 / 2^{\circ}$.

a discontinuity in the diffracted field in order to compensate. The complete (optics plus diffraction) field is, of course, continuous everywhere, but its oscillatory behavior in an angular region centered on the reflected wave boundary does lead to the corrections to the physical optics fields evident in the wedge approximation (23), and it is only at large distances from the edge, i.e., at points on the surface which are electrically remote from the nearest point on the reflected wave boundary, that these correction terms become negligible. It would appear that the same 
sort of mechanism is operating in the case of the cone, and the quantitative success of the wedge approximation suggests that the function which describes the perturbation field is closely related to the Fresnel integral. In this connection, the work of Felsen (1959), dealing with a ring source on the axis of a semiinfinite cone, and the extension by Bowman (1963) to the problem of a plane wave at axial incidence, are very relevant, although in neither case is the analysis applicable to the surface field as such.

The work described here was sponsored by the Air Force Avionics Laboratory of the Wright-Patterson Air Force Base, Ohio, under USAF Contract No. AF 33(615)-5170.

\section{References}

Bowman, J. J.-(1963), Far-zone scattering of plane electromagnetic waves by a perfectly conducting semi-infinite cone (nose-on incidence), Memorandum No. D0620-112-M, Conductron Corp., Ann Arbor, Mich.

Carrus, P. A., and C. G. Treuenfels (1950), Tables of roots and incomplete integrals of associated Legendre functions of fractional orders, J. Math. Phys. 29, 282-299.

Einarsson, O., and C. T. Tai (1967), The thin wire, (to be published).

Felsen, L. B. (1957), Alternative field representations in regions bounded by spheres, cones and planes, Trans. IRE-PGAP AP-5, No. 1, 109-121.

Felsen, L. B. (1959), Radiation from ring sources in the presence of a semi-infinite cone, Trans. IRE-PGAP AP-7, No. 2, 168-180.
Goodrich, R. F., and N. D. Kazarinoff (1963), Scalar diffraction by prolate spheroids whose eccentricities are almost one, Proc. Cambridge Phil. Soc. 59, 167-183.

Hansen, W. W., and L. I. Schiff (1948), Theoretical study of electromagnetic waves scattered from shaped metal surfaces, Stanford Univ. Microwave Lab. Repts. Nos. I through 4, Stanford, Calif. Magnus, W., and F. Oberhettinger (1949), Formulas and Theorems for the Special Functions of Mathematical Physics (Chelsea Publ. Co., New York, N.Y.).

Mentzer, J. R. (1955), Scattering and Diffraction of Radio Waves (Pergamon Press, New York, N.Y.).

National Bureau of Standards (1964), Handbook of Mathematical Functions with Formulas, Graphs, and Mathematical Tables, ed. M. Abramovitz and I. A. Stegun, NBS Appl. Math. Ser. 55.

Peters, L. (1956a), Memorandum on the echo areas of ogives, Antenna Lab. Rept. No. 601-7, Ohio State Univ., Columbus, Ohio.

Peters, L. (1956b), The end-fire echo area of long thin bodies, Antenna Lab. Rept. No. 601-9, Ohio State Univ., Columbus, Ohio. Peters, L. (1958), The end-fire echo area of long thin bodies, Trans. IRE-PGAP AP-6, No. 2, 133-139.

Siegel, K. M., J. W. Crispin, Jr., R. E. Kleinman, and H. E. Hunter (1952), The zeros of $P_{n_{i}}^{\prime}\left(x_{0}\right)$ of non-integral degree, J. Math. Phys. 31, 170-179.

Siegel, K. M., J. W. Crispin, Jr., R. E. Kleinman, and H. E. Hunter (1953), Note on the zeros of $d P_{m_{i}}^{\prime}(x) /\left.d x\right|_{x=x_{0}}$, J. Math. Phys. 32, $193-196$.

Vainshtein, L. A. (1959), Waves of current in a thin cylindrical conductor II, the current in a passive oscillator and the radiation of a transmitting antenna, Sov. Phys. Tech. Phys. 4, 617-626.

Waterman, P. C. (1963), Roots of Legendre functions of variable index, J. Math. Phys. 42, 323-328.

Zukowski, L. P., and T. B. A. Senior (1965), Surface field data for cone-sphere-like objects, Radiation Lab. Rept. No. 7030-6-T, Univ. of Michigan, Ann Arbor, Mich.

(Paper 2-5-215) 